\title{
Abdominal pelvic CT findings compared between COVID-19 positive and COVID-19 negative patients in the emergency department setting
}

\author{
Stacey A. Funt ${ }^{1} \cdot$ Stuart L. Cohen ${ }^{2,3} \cdot$ Jason J. Wang ${ }^{2,3} \cdot$ Pina C. Sanelli ${ }^{2,3} \cdot$ Matthew A. Barish $^{1} \mathbb{C}$ \\ Received: 2 July 2020 / Revised: 16 September 2020 / Accepted: 27 September 2020 / Published online: 12 October 2020 \\ (c) Springer Science+Business Media, LLC, part of Springer Nature 2020
}

\begin{abstract}
Purpose Manifestations of COVID-19 are primarily respiratory based, however, gastrointestinal symptoms are now recognized as an important component of the disease. The purpose of this study is to evaluate differences in abdominal pelvic CT findings in the emergency department by COVID-19 test result.

Methods This retrospective study identified patients tested by PCR for COVID-19 infection who underwent abdominal pelvic CT scan in the ED across an academic health system from March 15 to April 15, 2020. Radiology reports were reviewed for the presence of ground glass opacity in the lungs and acute abdominal pathology. A subset of patients with acute abdominal pathology were identified with inflammatory pathology in organs with high ACE2 receptor expression including bowel, pancreas, urinary bladder, and kidney. CT findings for COVID positive versus negative patients were compared with Chisquare test.

Results 597 patients tested by PCR for COVID-19 infection underwent abdominal pelvic CT scan, 44\% were COVID-19 positive. COVID-19 positive patients demonstrated significantly more ground glass opacity at the lung bases, $65.1 \%$, $(222 / 341)$ versus $12.4 \%$ (33/266), $p<0.001)$, and significantly less acute abdominal findings, $23.8 \%(81 / 341)$ versus $45.5 \%(121 / 266)$, $p \leq 0.001)$. When abdominal pathology was present, COVID-19 positive patients had higher rate of inflammatory pathology $58 \%(47 / 81)$ versus $29.8 \%(36 / 121)$.

Conclusions In patients undergoing abdominopelvic CT from the ED, COVID-19 positive patients are more likely to have ground glass opacities at the lung bases and less likely to have acute abdominal pathology compared with COVID-19 negative patients. Further, COVID-19 positive patients are more likely to have inflammation of organs with high expression of ACE2 receptors than other types of acute abdominal pathology.
\end{abstract}

Keywords COVID-19 - Abdominal pelvic CT · ACE2 receptor · Emergency radiology · Computed tomography · Gastrointestinal symptoms

Matthew A. Barish

mbarish@northwell.edu

1 Department of Radiology, Northwell Health, Manhasset, NY, USA

2 Department of Radiology, Imaging Clinical Effectiveness and Outcomes Research (ICEOR), Northwell Health, Manhasset, NY, USA

3 Center for Health Innovations and Outcomes Research (CHIOR), Feinstein Institutes for Medical Research and Donald and Barbara Zucker School of Medicine at Hofstra/Northwell, Manhasset, NY, USA

\section{Introduction}

COVID-19 pandemic has infected over 23.9 million people with over 821,000 deaths worldwide as of August 26, 2020 [1]. Many articles document COVID-19 imaging findings, yet most focus on thoracic abnormalities [2, 3]. However, COVID-19 has been shown to demonstrate multisystem and not just thoracic involvement [4].

Gastrointestinal symptoms including diarrhea, vomiting, and abdominal pain were initially reported at low rates, yet are now seen in $12-50 \%$ of patients and are considered an important aspect of the disease [5-9]. Lin et al. found 11.6\% of patients with COVID-19 and respiratory symptoms also had GI symptoms on admission, which increased to $49.5 \%$ during hospitalization. In one study, $6 \%$ of patients with 
COVID-19 presented with abdominal symptoms without any respiratory abnormalities [10]. Virus shedding has been detected in feces in over $40 \%$ of infected patients [11].

Imaging studies for acute non-respiratory symptoms in COVID-19 patients are often negative. Hossain et al. reported no acute abdominal findings in (68\%) 69/101 patients of COVID-19 patients on abdominal pelvic CT scan. When abdominal pathology is present, the bowel is often affected. Goldberg-Stein et al. reported abdominal pelvic pathology in 57\% of COVID-19 positive patients with $31 \%$ showing GI tract abnormalities [12]. Bhayana et al. found $31 \%$ of CT scans had bowel pathology in patients with COVID-19. The authors note a high level of Angiotensinconverting enzyme 2 (ACE2) receptors in the bowel, and offer this as a possible explanation of these findings [13]. SARS-CoV-2 is known to enter the host cell via these receptors. While these were initially described in the lung, organs elsewhere in the body are now known to have high expression. In addition to bowel, organs in the abdomen described to have high expression of ACE2 receptors include the pancreas, kidneys, and bladder [14-20].

In the emergency department, clinical decisions regarding the imaging of patients must be made prior to the availability of COVID-19 PCR test results. In some cases, patients presenting with abdominal symptoms may not undergo PCR testing prior to imaging. Radiologists have been the first to suggest the diagnoses of COVID-19 infection based on the presence of ground glass opacities at the lung bases in patients presenting with abdominal symptoms [21]. In addition, we postulated that COVID-19 positive patients might demonstrate acute pathology in the abdomen that differs from COVID-19 negative patients, possibly due to the presence of high ACE2 receptor expression in certain organs. Therefore, patients presenting with significant abdominal symptoms warranting abdominal pelvic $\mathrm{CT}$ scan during the COVID-19 pandemic are an important cohort to evaluate. To our knowledge, no study has directly compared abdominal pelvic CT scan findings for COVID-19 positive and negative patients. Our goal was to determine if patients who ultimately tested positive for COVID-19 infection had different rates and types of abdominal pathology reported on abdominal pelvic CT scans.

\section{Materials and methods}

\section{Study design, setting, and population}

This retrospective study evaluated consecutive adult patients tested for COVID-19 infection who underwent abdominal pelvic CT scan in the emergency department across an academic health system from March 15 to April 15, 2020.
Patients were excluded if they were not tested for COVID-19 within $24 \mathrm{~h}$ of the CT or the study was performed for evaluation of trauma. If a patient underwent multiple CT studies, only the initial study was evaluated. The hospital system includes 12 hospital, 5 which are tertiary and 7 of which are community based. Dates were chosen to correspond to early onset to the peak of the COVID pandemic in our region. The study was performed with approval of the institutional review board and waiver of consent.

\section{Data variables and collection}

All CT studies of the abdomen and pelvis (with contrast, without contrast, and without/with contrast) performed within the study timeframe were identified by searching the radiology information system (RIS). Demographic information (age, gender, race) and COVID-19 PCR testing status were extracted from the system electronic health record system for all patients included in the study. COVID-19 status was determined by nasal swab PCR result in the ED or during the hospital admission. All facilities used the same PCR test. The COVID-19 tests could have been acquired $24 \mathrm{~h}$ before or after the CT.

\section{Radiology report analysis}

Radiology reports from the abdominal pelvic CT scans were evaluated for the presence of ground glass opacities (GGO) at the lung bases, acute abdominal pathology, including a subset of inflammatory pathology. The impression of the radiology report was reviewed to characterize CT findings and when unclear, the entire report and/ or images were evaluated. Evaluation of all reports and studies were performed by a single board-certified radiologist fellowship trained in body imaging with 24 years of experience (SF) blinded to clinical data outside of the radiology report.

The presence of GGO at the lung bases was considered based on report impression, and if unclear, full radiology report and/or image evaluation. If a concomitant chest CT scan was reported, only the results of the lung bases were considered. Focal consolidation reported as bacterial pneumonia or atelectasis were not considered GGO.

Intraabdominal pathology was classified as acute abdominal pathology ("abdominal pathology") or as the absence of acute abdominal pathology ("no abdominal pathology"). A subset of patients with acute abdominal pathology were identified with inflammatory pathology in organs with previously reported high ACE2 receptor expression including bowel, pancreas, urinary bladder, and kidney. The presence or absence and type of acute abdominal pathology was considered based on report 
impression. Acute abdominal pathology was defined by any inflammatory, infectious or obstructing etiologies, thromboses, hemorrhage, and neoplasm that could cause acute symptoms. Chronic or asymptomatic conditions such as hepatic steatosis, post-surgical biliary dilation, cirrhosis, diverticulosis, non-obstructing stones, neoplasm, and degenerative changes in bone were not considered to be acute pathology. In cases where the impression was vague, the report was used to help clarify the impression. For the few cases where the report was non-specific or vague, the images were viewed directly by one of the radiologists (SF) to determine the presence/absence of acute abdominal pathology.

\section{Statistical analysis}

GGO, acute abdominal pathology, and inflammatory pathology in organs with high ACE2 receptor expression were compared by COVID-19 result status for all patients. Further, the presence of abdominal pathology and inflammatory pathology in organs with high ACE2 receptor expression was compared by COVID-19 and GGO for all patients and stratified by COVID and GGO findings. Statistical analyses were performed using Chi square. Statistical significance was considered for $p$ value $<0.05$. All statistical analyses were done in SAS v9.4 (SAS Institute).

\section{Results}

Our initial search revealed 2375 ED patients who underwent CT of the abdomen and pelvis within the study timeframe. Patient cohort consisted of 597 patients (Fig. 1): 292 (48.9\%) men and 305 (51.1\%) women in age groups 19-39 (17.6\%), 40-59 (33.5\%), 60-79 (34.3\%), and 80+ (14.6\%) (Table 1). A total of 23/597 (3.9\%) of cases had the images directly reviewed. Results of COVID-19 PCR testing were negative in 259 patients (43.4\%) and positive in 338 patients (56.6\%). There were no significant differences in gender, age, race, study location, or use of IV contrast by COVID result.

Findings on abdominal pelvis CT scan are summarized in Tables 2, 3 and Figs. 2, 3, 4, 5, and 6. The absence of abdominal pathology was more common in COVID-19 positive patients $(258 / 338,76.3 \%)$ compared with COVID-19 negative patients $(140 / 259,54.1 \%)(p<0.001)$. Additionally, the absence of abdominal pathology was more common in patients with chest CT $(109 / 144,75.7 \%)$ compared with patients without chest CT $(289 / 453,63.8 \%)(p=0.027)$. However, when acute intraabdominal pathology was detected, COVID-19 positive patients were more likely to have inflammation in an organ with high ACE2 expression compared with COVID-19 negative patients (47/80, 58.8\%
2,375 Patients with CT $\mathrm{Abd} /$ Pelvis in ED from March 15-April 152020

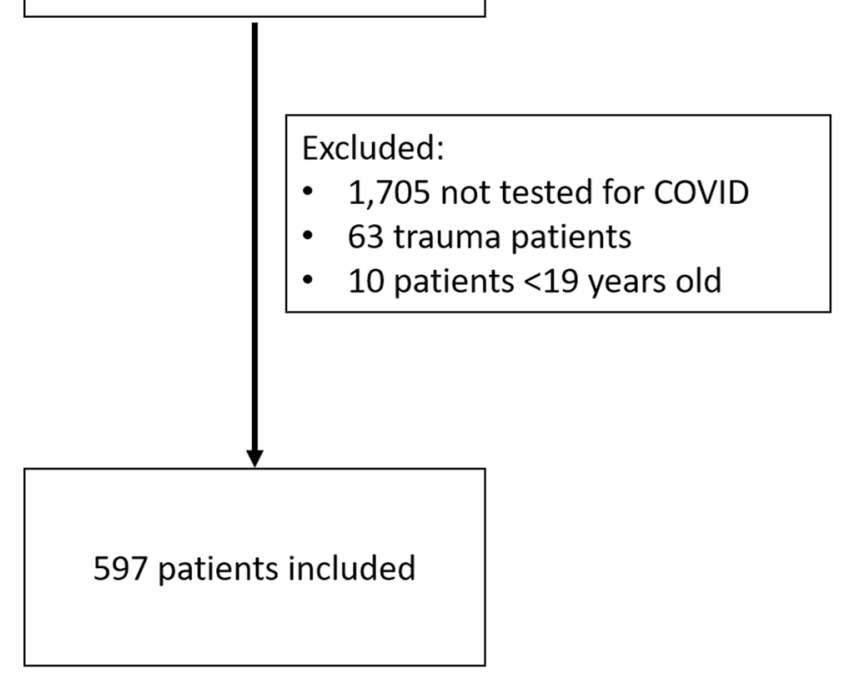

Fig. 1 Study design and patients

Table 1 Patient characteristics

\begin{tabular}{|c|c|c|c|c|}
\hline $\begin{array}{l}\text { Patient } \\
\text { characteristics }\end{array}$ & $\begin{array}{l}\text { Overall } \\
N=597\end{array}$ & $\begin{array}{l}\text { COVID- } \\
N=259\end{array}$ & $\begin{array}{l}\text { COVID+ } \\
N=338\end{array}$ & $p$ value \\
\hline \multicolumn{5}{|l|}{ Sex } \\
\hline Male & $292(48.9 \%)$ & $126(48.6 \%)$ & $166(49.1 \%)$ & \multirow[t]{2}{*}{0.911} \\
\hline Female & $305(51.1 \%)$ & $133(51.4 \%)$ & $172(50.9 \%)$ & \\
\hline \multicolumn{5}{|l|}{ Age } \\
\hline $19-39$ & $105(17.6 \%)$ & $53(20.5 \%)$ & $52(15.4 \%)$ & \multirow[t]{4}{*}{0.223} \\
\hline $40-59$ & $200(33.5 \%)$ & $88(34.0 \%)$ & $112(33.1 \%)$ & \\
\hline $60-79$ & $205(34.3 \%)$ & $87(33.6 \%)$ & $118(34.9 \%)$ & \\
\hline $80+$ & $87(14.6 \%)$ & $31(11.2 \%)$ & $56(16.6 \%)$ & \\
\hline \multicolumn{5}{|l|}{ Race } \\
\hline Asian & $37(6.2 \%)$ & $17(6.6 \%)$ & $20(5.9 \%)$ & \multirow[t]{4}{*}{0.091} \\
\hline $\begin{array}{l}\text { African Ameri- } \\
\text { can }\end{array}$ & $82(13.7 \%)$ & $30(11.6 \%)$ & $52(15.4 \%)$ & \\
\hline Other/unknown & $195(32.6 \%)$ & $75(29 \%)$ & $120(35.5 \%)$ & \\
\hline White & $288(47.4 \%)$ & $137(52.9 \%)$ & $146(43.2 \%)$ & \\
\hline \multicolumn{5}{|l|}{ Study location } \\
\hline Tertiary & $402(67.3 \%)$ & $173(66.8 \%)$ & $229(69 \%)$ & \\
\hline Community & $195(32.7 \%)$ & $86(33.2 \%)$ & $103(31 \%)$ & \\
\hline \multicolumn{5}{|l|}{ IV contrast } \\
\hline Yes & $457(76.6 \%)$ & $206(79.5 \%)$ & $251(74.3 \%)$ & \multirow[t]{2}{*}{0.132} \\
\hline No & $140(23.5 \%)$ & $53(20.5 \%)$ & $87(25.7 \%)$ & \\
\hline \multicolumn{5}{|l|}{ COVID status } \\
\hline Positive & $338(56.6 \%)$ & NA & NA & \multirow[t]{2}{*}{ NA } \\
\hline Negative & $259(43.4 \%)$ & NA & NA & \\
\hline \multicolumn{5}{|l|}{ Chest CT } \\
\hline No & $453(75.6 \%)$ & $196(75.1 \%)$ & $257(75.6 \%)$ & \multirow[t]{2}{*}{0.919} \\
\hline Yes & $144(24 \%)$ & $63(24.1 \%)$ & $81(23.8 \%)$ & \\
\hline
\end{tabular}


and $35 / 119,29.4 \%$, respectively, $p<0.001)$. However, when acute intraabdominal pathology was detected, there was no difference between patients with versus without chest $\mathrm{CT}$ in the presence of inflammation in an organ with high ACE2, (16/35) 45.7\% versus (66/164) 40.2\%, respectively. Among patients with inflammation in organs with high ACE2 receptors, the distribution of what organs were involved was the same between those patients with and without COVID, and with and without chest CT ( $p=0.904$ and $p=0.144$, respectively).

There was also no significant difference in the rate of acute abdominal pathology by COVID-19 status in patients with ground glass opacities $(p=0.094)$, yet in the subset of patients without ground glass opacity at the lung bases, the absence of abdominal pathology in COVID-19 positive patients was seen more frequently $(82 / 117,70.1 \%)$ compared with COVID-19 negative patients $(118 / 226,52.2 \%)$ $(p=0.002)$.
As expected, ground glass opacities were more commonly seen at the lung bases in COVID-19 positive patients $(221 / 338,65.4 \%)$ than in COVID-19 negative patients $(33 / 259,12.7 \%)(p<0.001)$.

\section{Discussion}

Ground glass opacities are the most common lung finding in COVID-19 seen in $56.4 \%$ of chest CT scans [6]. In our study, we found $65.4 \%$ of COVID-19 positive patients had ground glass opacities at the lung bases on abdominal pelvic CT scan which was a significant difference from COVID19 negative patients $(12.7 \%)$. This prevalence of ground glass opacity in COVID-19 positive patients has been corroborated elsewhere. In a recent paper by Hossain et al., the authors found that $63.9 \%$ of patents presenting to the ED with neurologic or abdominal symptoms had GG opacities in the lungs [4]. In our experience, especially at the onset

Table 2 Abdominal CT scan findings

\begin{tabular}{|c|c|c|c|c|c|c|c|}
\hline Findings & All patients & COVID- & COVID+ & $p$ value & Without chest CT & With chest $\mathrm{CT}$ & $p$ value \\
\hline \multicolumn{8}{|l|}{ Ground glass opacity } \\
\hline GGO- & $343(57.5 \%)$ & $226(87.3 \%)$ & $117(34.6 \%)$ & \multirow[t]{3}{*}{$<0.001$} & $271(59.8 \%)$ & $72(50 \%)$ & \multirow[t]{3}{*}{0.038} \\
\hline GGO+ & $254(42.5 \%)$ & $33(12.7 \%)$ & $221(65.4 \%)$ & & $182(40.2 \%)$ & $72(50 \%)$ & \\
\hline Total & 597 & 259 & 338 & & 453 & 144 & \\
\hline \multicolumn{8}{|l|}{ Abdominal pelvic CT findings in all patients } \\
\hline $\begin{array}{l}\text { Inflammation in organs with high ACE2 } \\
\text { receptors }\end{array}$ & $82(13.7 \%)$ & $35(13.5 \%)$ & $47(13.9 \%)$ & \multirow[t]{4}{*}{$<0.001$} & $66(14.6 \%)$ & $16(11.1 \%)$ & \multirow[t]{4}{*}{0.027} \\
\hline Other acute abdominal pathology & $117(19.6 \%)$ & $84(32.4 \%)$ & $33(9.8 \%)$ & & $98(21.6 \%)$ & $19(13.2 \%)$ & \\
\hline No abnormal findings & $398(66.7 \%)$ & $140(54.1 \%)$ & $258(76.3 \%)$ & & $289(63.8 \%)$ & $109(75.7 \%)$ & \\
\hline Total & 597 & 259 & 338 & & 453 & 144 & \\
\hline \multicolumn{8}{|c|}{ Type of abdominal pathology in those patients with acute findings } \\
\hline $\begin{array}{l}\text { Inflammation in organs with high ACE2 } \\
\text { receptors }\end{array}$ & $82(41.2 \%)$ & $35(29.4 \%)$ & $47(58.8 \%)$ & \multirow[t]{3}{*}{$<0.001$} & $66(40.2 \%)$ & $16(45.7 \%)$ & \multirow[t]{3}{*}{0.551} \\
\hline Other acute abdominal pathology & $117(58.8 \%)$ & $84(70.6 \%)$ & $33(41.3 \%)$ & & $98(59.8 \%)$ & $19(54.3 \%)$ & \\
\hline Total & 199 & 119 & 80 & & 164 & 35 & \\
\hline \multicolumn{8}{|c|}{ Inflammation in organs with high ACE2 expression } \\
\hline Bowel Inflammation & $38(46.3 \%)$ & $15(42.9 \%)$ & $23(48.9 \%)$ & \multirow[t]{5}{*}{0.904} & $28(42.4 \%)$ & $10(62.5 \%)$ & \multirow[t]{5}{*}{0.144} \\
\hline Pancreatitis & $9(11 \%)$ & $4(11.4 \%)$ & $5(10.6 \%)$ & & $6(9.1 \%)$ & $3(18.8 \%)$ & \\
\hline Pyelonephritis & $8(9.8 \%)$ & $3(8.6 \%)$ & $5(10.6 \%)$ & & $8(12.1 \%)$ & $0(0 \%)$ & \\
\hline Cystitis & $27(32.9 \%)$ & $13(37.1 \%)$ & $14(29.8 \%)$ & & $24(36.4 \%)$ & $3(18.8 \%)$ & \\
\hline Total & 82 & 35 & 47 & & 66 & 16 & \\
\hline \multicolumn{8}{|l|}{ Other abdominal pathology details } \\
\hline Bowel obstruction & $17(14.5 \%)$ & $15(17.9 \%)$ & $2(6.1 \%)$ & \multirow[t]{7}{*}{0.439} & $16(16.3 \%)$ & $1(5.3 \%)$ & \multirow[t]{7}{*}{0.128} \\
\hline Acute cholecystitis & $9(7.7 \%)$ & $7(8.3 \%)$ & $2(6.1 \%)$ & & $7(7.1 \%)$ & $2(10.5 \%)$ & \\
\hline Hydronephrosis & $20(17.1 \%)$ & $14(16.7 \%)$ & $6(18.2 \%)$ & & $19(19.4 \%)$ & $1(5.3 \%)$ & \\
\hline Acute appendicitis & $5(4.3 \%)$ & $4(4.8 \%)$ & $1(3 \%)$ & & $5(5.1 \%)$ & $0(0 \%)$ & \\
\hline Diverticulitis & $7(6 \%)$ & $4(4.8 \%)$ & $3(9.1 \%)$ & & $7(7.1 \%)$ & $0(0 \%)$ & \\
\hline Other & $59(50.4 \%)$ & $40(47.6 \%)$ & $19(57.6 \%)$ & & $44(44.9 \%)$ & $15(78.9 \%)$ & \\
\hline Total & 117 & 84 & 33 & & 98 & 19 & \\
\hline
\end{tabular}




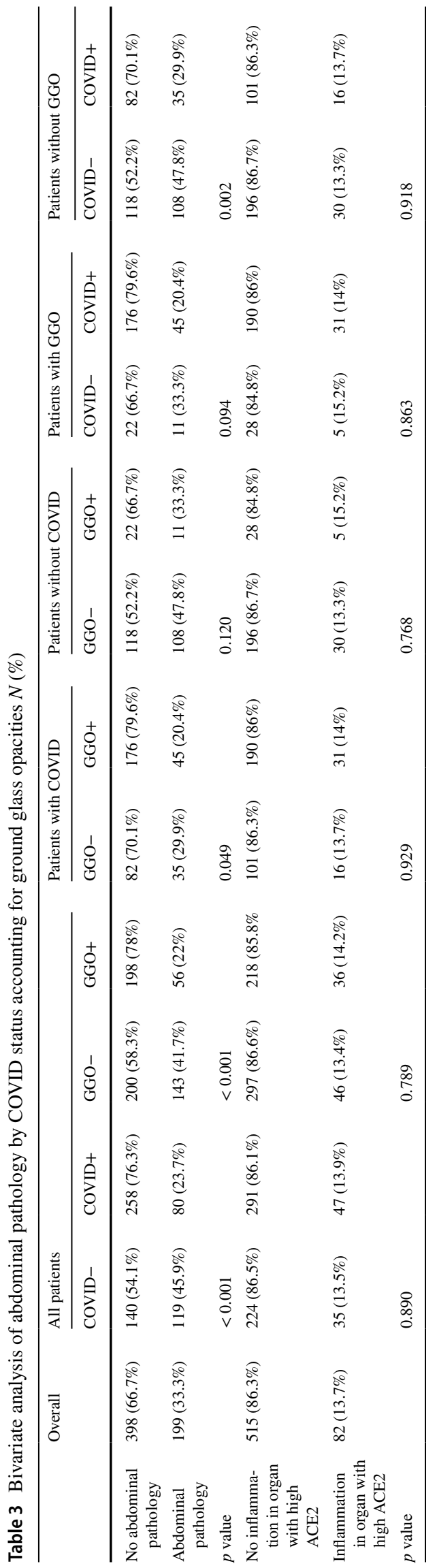

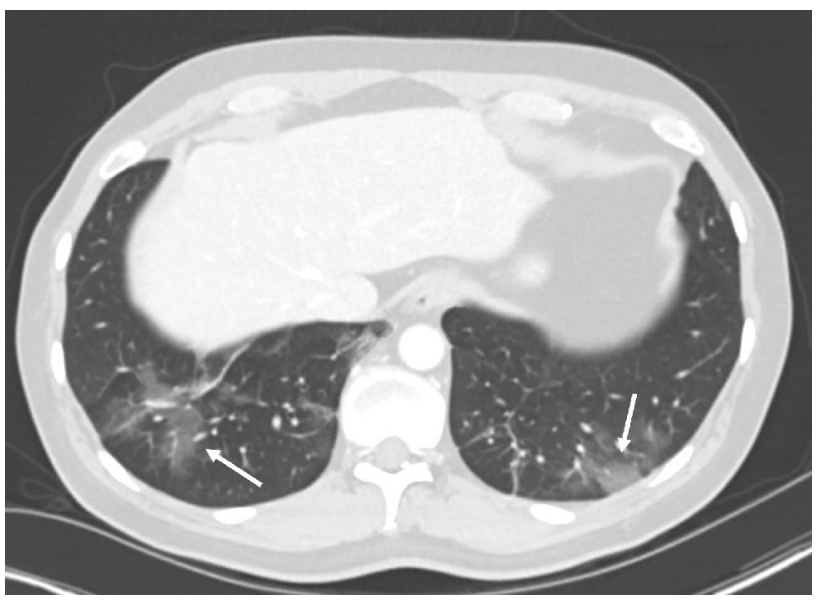

Fig. 2 A 50-year-old male, COVID-19 PCR positive, presented with bilateral lower lobe ground glass opacities (arrows) with no intraabdominal pathology reported (not shown)

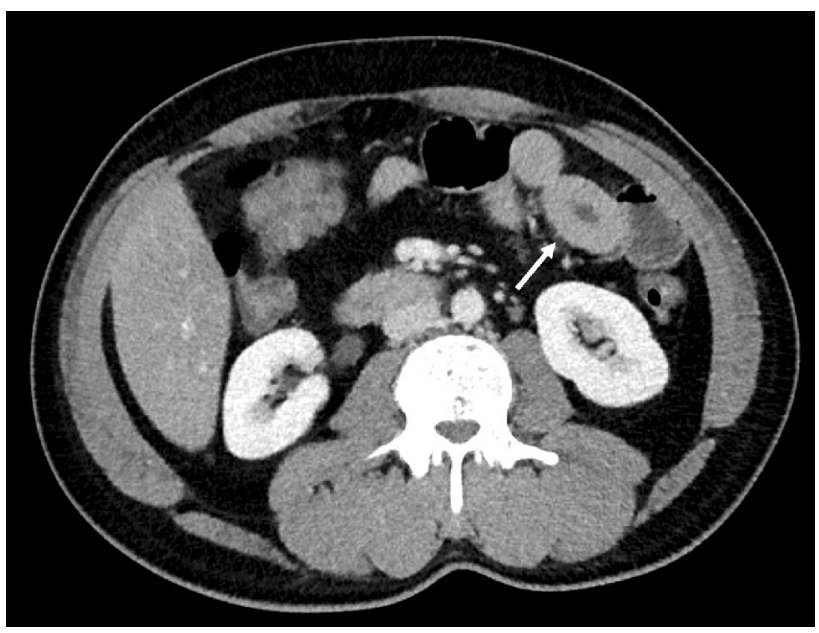

Fig. 3 A 49-year-old male, COVID-19 PCR positive, presented with left-sided abdominal pain, with thick-walled loop of small bowel (arrow) with mild peri-enteric fat stranding, suggesting enteritis

of the pandemic before gastrointestinal manifestations were widely recognized as a presenting symptom, the radiologist was often first to suggest the diagnosis of COVID-19 when ground glass opacities were visualized on abdominal pelvic CT.

Another significant finding in this study is that COVID19 negative patients had more acute pathologic findings on abdominopelvic CT scan than COVID-19 positive patients. We theorize that patients who were COVID positive who underwent $\mathrm{CT}$ were more likely to have milder disease due to generalized COVID infection. However, patients without COVID infection with milder symptoms did not seek medical care and were not included in this study. Thus, the 


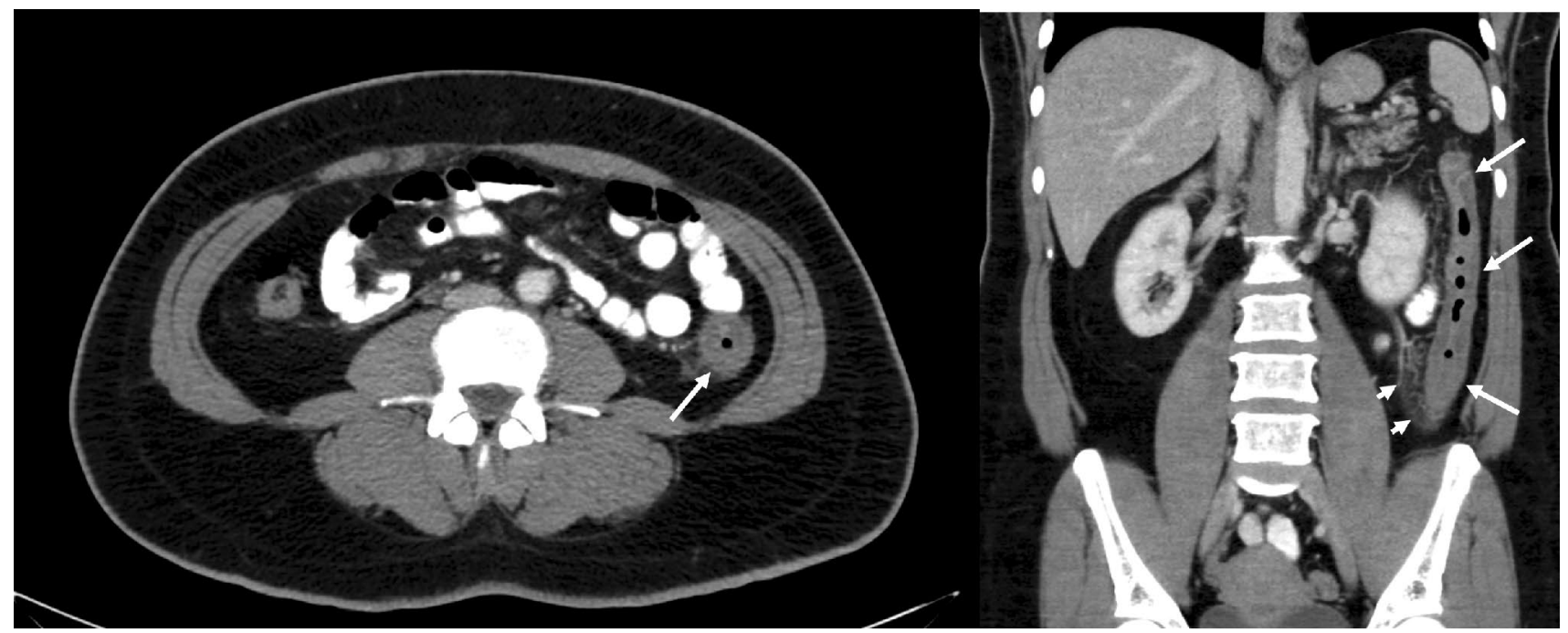

Fig. 4 A 53-year-old female, COVID-19 PCR positive, presented with pelvic pain, with thick-walled loop of descending colon (arrow) with mild peri-enteric fat stranding (short arrows), representing colitis

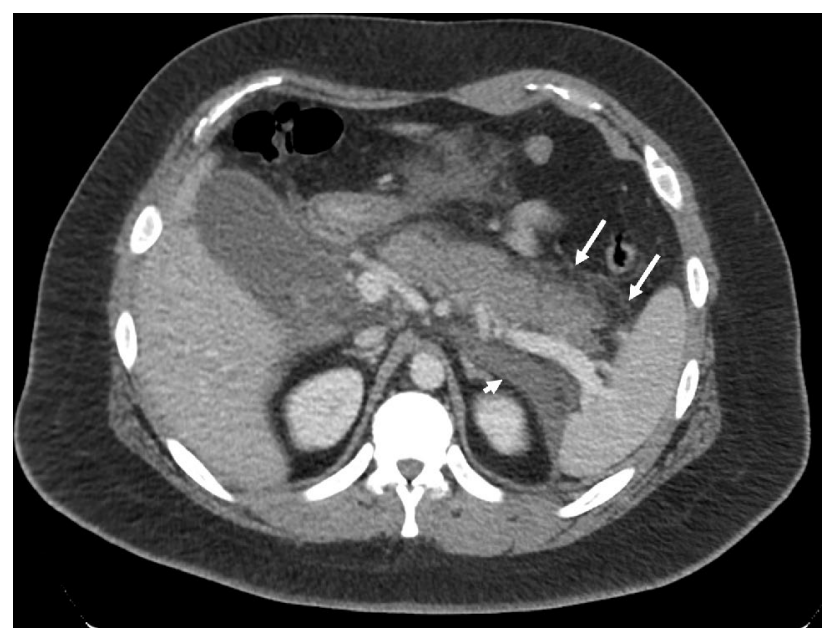

Fig. 5 A 23-year-old male, COVID-19 PCR positive, presented with abdominal pain, with pancreatic enlargement, peri-pancreatic edema (arrows), and fluid in left pararenal space (short arrows) consistent with acute pancreatitis

positive rate in COVID-19 negative patients who did seek medical care increased.

Another possible explanation for this finding is the welldocumented communication between the lung and the gut called the "gut-lung" axis [10, 22, 23]. In this bidirectional system, microbial metabolites and endotoxins released into the blood from lung inflammation can alter the microbiome of the gut leading to abdominal symptoms in the absence of intraabdominal pathology. In our study, we also found that patients without ground glass opacities at the lung bases had less acute intraabdominal pathology, if they were COVID-19 positive. This may be due to non-visualized infection in the

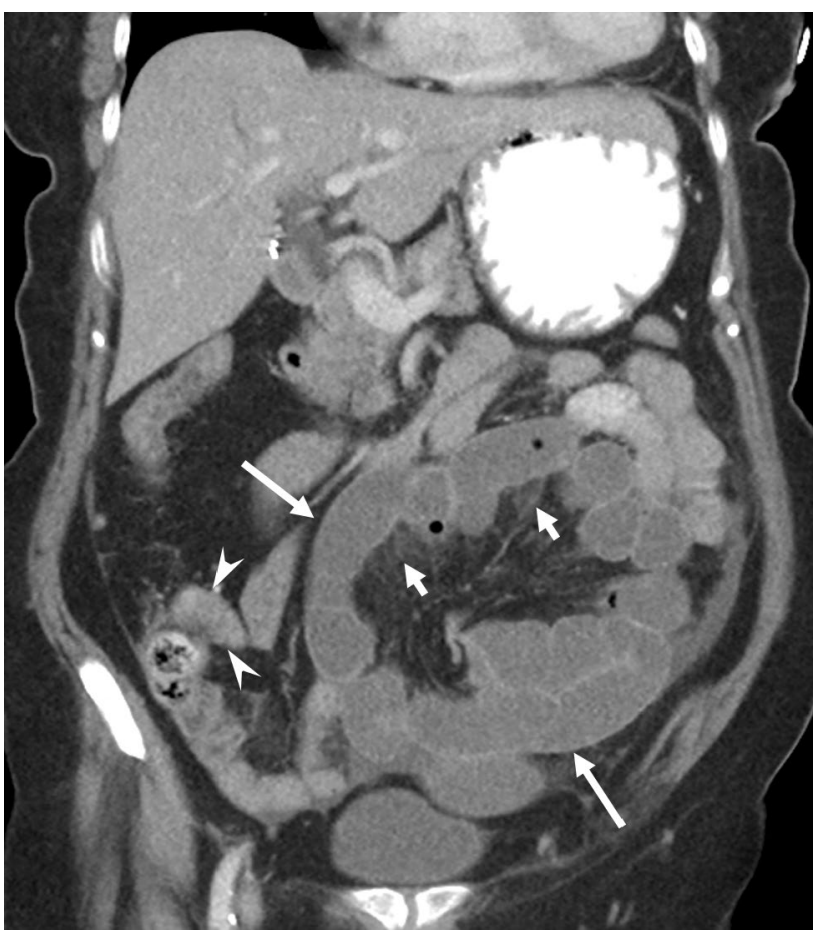

Fig. 6 A 75-year-old female, COVID-19 PCR negative, presented with abdominal pain, with mesenteric edema (short arrows), dilated ileum (arrows), and decompressed terminal ileum (arrow heads) consistent with small bowel obstruction

mid and upper lungs also affecting the gut via the gut-lung axis producing secondary abdominal symptoms. Alternatively, this may be due to the limited ability of abdominal pelvic CT scan to detect mild inflammation or be compounded by the lack of IV contrast in $23.5 \%$ of studies. We 
did not find a statistical difference between COVID-19 negative and positive patients' rate of abdominal pathology when ground glass opacities were present. This might be explained by possible lung inflammation in the COVID-19 negative group from causes other than COVID-19 also affecting the gut via the same axis.

In our study, we tracked the most common acute diagnoses mentioned in the impression of the radiology report that could account for abdominal pain and compared COVID19 positive and negative patients. In the subset of patients that had acute pathology, COVID-19 positive patients had bowel inflammation, pancreatitis, pyelonephritis, or cystitis more often than COVID-19 negative patients. Of those with acute abdominal pathology, our most common diagnosis was bowel inflammation seen in (23/80) $28.8 \%$ of COVID19 positive cases. This is similar to the $31 \%$ reported by Bhayana et al. This trend was not seen when we looked at COVID-19 negative patients. One possible explanation is that organs with high ACE 2 receptor expression may be at increased risk for tissue injury and inflammation by SARSCoV-2. This virus is also known to cause liver injury [24], although periportal edema or heterogeneously enhancing parenchyma was not documented in any report impressions in our study. There is also increased ACE 2 receptor expression in the biliary tree [25], although we did not have any cases of cholangitis. This may be due to the poor sensitivity of $\mathrm{CT}$ for this diagnosis.

A limitation of our study is that we relied primarily on the original radiology report impressions to compile our data without reinterpretation of the images. This may be an opportunity for future research. There can be variability between radiologist's interpretations and whether a diagnosis is reported as definitive or qualified in some manner. Additionally, the radiology reports were analyzed by a single reader. In addition, we did not analyze our results with respect to IV or oral contrast. Nonetheless, we had the same limitations in both of our groups. Another limitation is the variability of the RT-PCR test results which includes the possibility of false-positive and false-negative results. An additional limitation was the inability to account for hepatic steatosis. This was because of the variability in reporting and variability in use of IV contrast. This is an important area for future research. Inclusion of only ED patients who underwent abdominal imaging excluded patients who underwent imaging in the outpatient setting. Further, the etiology of the underlying process of the inflammatory process was not determined in this study. As such, we did not attempt to differentiate between inflammation secondary to bacterial infection, viral infection, or other non-infectious etiology. Finally, this study determines correlation and not causality between COVID-19 and organ involvement of disease.

\section{Conclusion}

In patients undergoing abdominopelvic $\mathrm{CT}$ from the $\mathrm{ED}$, COVID-19 positive patients are more likely to have ground glass opacities at the lung bases and less likely to have acute abdominal pathology compared with COVID-19 negative patients. Further, COVID-19 positive patients are more likely to have inflammation of organs with high expression of ACE2 receptors than other types of acute abdominal pathology.

\section{References}

1. Johns Hopkins Coronavirus Resource Center https://coronaviru s.jhu.edu/map.html. Assess August 26, 2020.

2. Salehi S, Abedi A, Balakrishnan S, Gholamrezandezhad a. Coronavirus Disease 2019 (COVID-19): A Systemic Review of Imaging Findings in 919 Patients. AJR Am J Roentgenol. 2020 Jul;215(1):87-93. https://doi.org/10.2214/ajr.20.23034. Epub 2020 Mar 14.

3. Zu ZY, Jiang MD, Xu PP, Chen W, Ni QQ, Lu GM, Zhang LJ. Coronavirus Disease 2019 (COVID-19): A Perspective from China. Radiology published online Feb 4, 2020. https://doi. org/10.1148/radiol.2020200230

4. Hossain R, Lazarus M, Roudenko A, Dako F, Mehta V, Alis J, Zalta B, Lei B, Haramati L, White C. CT Scans Obtained for Nonpulmonary Indications: Associated Respiratory Findings of COVID-19. Thoracic Imaging. Published Online: May 112020 https://doi.org/10.1148/radiol.2020201743

5. Huang C, Wang Y, Li X, et al. Clinical features of patients infected with 2019 novel Coronarius in Wuhan, Lancet. 2020;395:497-506. https://doi.org/10.1016/s0140 -6736(20)30183-5pmid: http://www.ncbi.nlm.nih.gov/pubme $\mathrm{d} / 31986264$

6. Guan W-J, Ni Z-Y, Hu Y et al. Clinical Characteristics of Coronavirus Disease 2019 in China. N Engl J Med. 2020;http://dx.doi. org/10.1056/NEJMoa2002032

7. Siew C N, Herbert T. COVID-19 and the gastrointestinal tract: more than meets the eye. Gut, 2020 - gut.bmj.com. http://dx.doi. org/10.1136/gutjnl-2020-321195

8. Jin X, Lian J-S, Hu J-H, et al. Epidemiological, clinical and virological characteristics of 74 cases of coronavirus-infected disease 2019 (COVID-19) with gastrointestinal symptoms. Gut 2020. doi:https://doi.org/10.1136/gutjnl-2020-320926. [Epub ahead of print: 24 Mar 2020].pmid:http://www.ncbi.nlm.nih.gov/pubme $\mathrm{d} / 32213556$

9. Lin L, Jiang X, Zhang Z, et al. Gastrointestinal symptoms of 95 cases with SARS-CoV-2 infection. Gut 2020. doi:https://doi. org/10.1136/gutjnl-2020-321013. [Epub ahead of print: 02 Apr 2020].pmid:http://www.ncbi.nlm.nih.gov/pubmed/32241899

10. Pan L, Mu M, Yang P, et al. Clinical characteristics of COVID-19 patients with digestive symptoms in Hubei, China: a descriptive, cross-sectional, multicenter study. Am J Gastroenterol. 2020. https ://journals.1ww.com/ajg/Documents/COVID_Digestive_Sympt oms_AJG_Preproof.pdf. March 18, 2020

11. Parasa A, Madhav D, Chandrasekar VT. Prevalence of Gastrointestinal Symptoms and Fecal Viral Shedding in Patients with Coronavirus Disease 2019. A Systemic Review and Meta-analysis. JAMA June 11, 2020. Netw Open. 2020;3(6):e2011335. https ://doi.org/10.1001/jamanetworkopen.2020.11335 
12. Goldberg-Stein A, Fink A, Paroder V, Kobi M, Yee J, Chernyak V. Abdominopelvic CT findings in patients with novel coronavirus disease 2019 (COVID-19). Abdom Radiol (NY). 2020 Aug 6:1-11. https://doi.org/10.1007/s00261-020-02669-2 [Epub ahead of print]

13. Bhayana R, et al. Abdominal Imaging Findings in COVID-19: Preliminary Observations. Radiology. Published Online:May 11 2020 https://doi.org/10.1148/radiol.2020201908

14. Ong J, Young B E, Ong S. Covid-19 in Gastroenterology: A Clinical Perspective. Gut Published Online First: 20 March 2020. doi: https://doi.org/10.1136/gutjnl-2020-321051

15. Liang W, Feng Z, Rao S, et al. Diarrhoea may be underestimated: a missing link in 2019 novel coronavirus. Gut 2020:gutjnl-2020-320832.doi:10.1136/gutjn1-2020320832pmid:http://www.ncbi.nlm.nih.gov/pubmed/32102928

16. Luo S, Zhang X, Xu H. Don't Overlook Digestive Symptoms in Patients With 2019 Novel Coronavirus Disease (COVID-19). Clin Gastroenterol Hepatol. 2020;http://dx.doi.org/10.1016/j. cgh.2020.03.04

17. Wang F, Wang H, Fan J, Shang Y, Wang H, Zhao Q. Pancreatic injury patterns in patients with COVID-19 pneumonia. Gastroenterology. 2020 Apr 1. pii: S0016-5085(20)30409-1. https://doi. org/10.1053/j.gastro.2020.03.055. [Epub ahead of print]

18. Zou X, Chen K, Zou J, Han P, Hao J, Han Z. Single-cell RNAseq data analysis on the receptor ACE2 expression reveals the potential risk of different human organs vulnerable to 2019-nCoV infection. Front Med. 2020;http://dx.doi.org/10.1007/s11684-020

19. Furong Liu, Xin Long, Wenbin Zou, Minghao Fang, Wenjuan Wu, Wei Li, Bixiang Zhang, Wanguang Zhang, Xiaoping Chen, Zhanguo Zhang. Highly ACE2 Expression in Pancreas May Cause Pancreas Damage After SARS-CoV-2 Infection. MedRxiv. https ://doi.org/10.1101/2020.02.28.20029181

20. Muff JN, Osterman A, Ruzicka M, Stihl C, Vilsmaler T, Munker D, Khatamzas E, Giessen-Jung C, Stief C, Steckler M, Severin
R. Urinary Frequency as a Possibly Overlooked Symptom in COVID-19 Patients: Does SARS-CoV-2 Cause Viral Cystitis? European Urology. Online 5/19/202. https://doi.org/10.1016/j. eururo.2020.05.013

21. Siegel A, Chang P, Jarou Z, Paushter D, Harmath C, Arevalo B, Dachman A. Lung Base Findings of Coronavirus Disease (COVID-19) on Abdominal CT in Patients With Predominant Gastrointestinal Symptoms. American Journal of Roentgenology: 1-3. https://doi.org/10.2214/ajr.20.23232. https://www.ajronline. org/doi/full/10.2214/AJR.20.23232

22. Dhar D, Mohanty A. Gut microbiota and Covid-19- possible link and implications. Virus Res. 2020 May 13 : 198018. https://doi. org/10.1016/j.virusres.2020.198018

23. Budden KF, Gellarly SL, Wood DLA, Cooper M, Morrison M, Hugenholtz P, Hansboro P. Emerging Pathogenic Links Between Microbiota and the Gut-Lung Axis. Nature Reviews Microbiology 15, 55-63 (2017)

24. Zhang C, Shi L, Wang F-S. Liver injury in COVID-19: management and challenges. The Lancet Gastroenterology \& Hepatology. 2020.

25. Chai, X. Hu L, Zhang Y, Han W, Lu Z, Ke A, Zhou J, She G, Fang J, Fan J, Cai J, Fan J, Lan F. Specific ACE2 Expression in Cholangiocytes May Cause Liver Damage After 2019nCoV Infection. Preprint at https://www.biorxiv.org/conte $\mathrm{nt} / 10.1101 / 2020.02 .03 .931766 \mathrm{v} 1$ (2020).

Publisher's Note Springer Nature remains neutral with regard to jurisdictional claims in published maps and institutional affiliations. 